\title{
Daten und Abbildungen aus dem Zahlenspiegel der Heinrich-Heine-Universität Düsseldorf
}





\section{Organigramm}

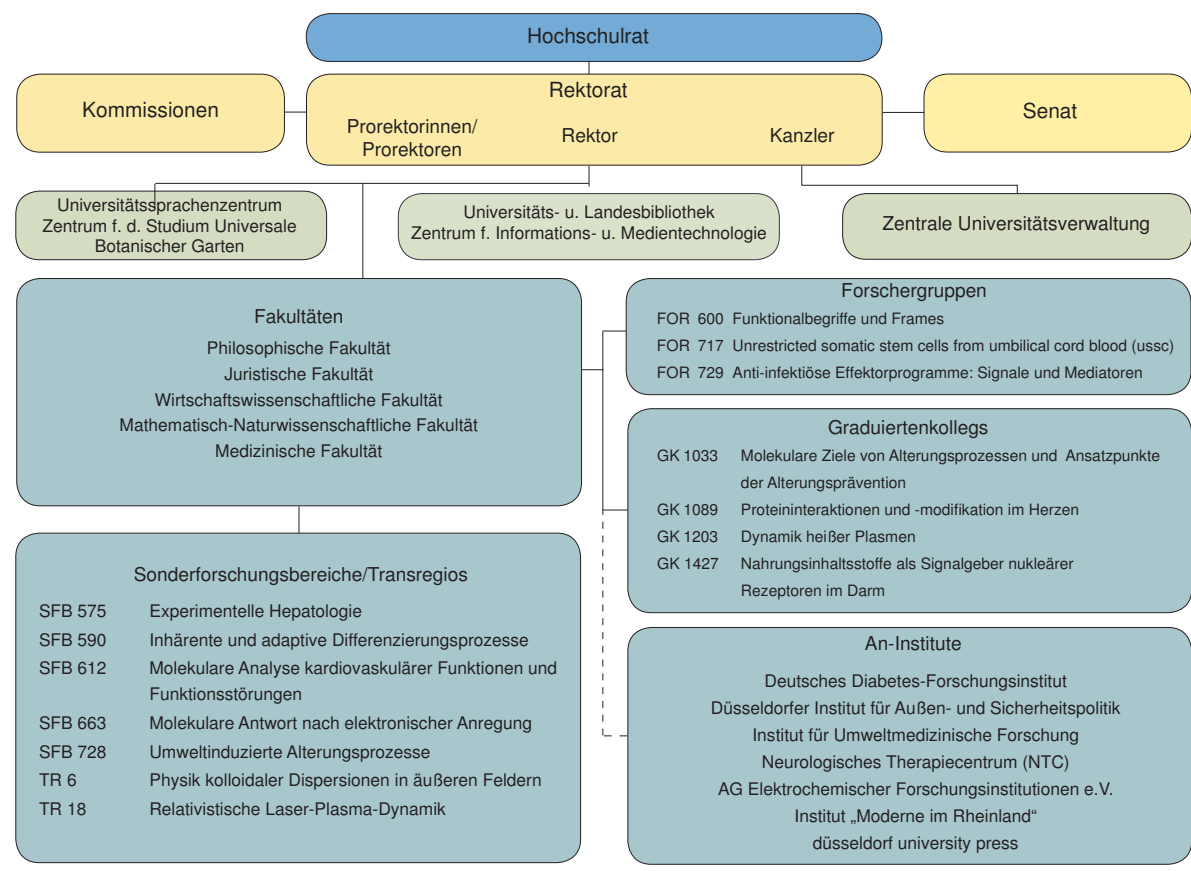

\section{Hochschulpartnerschaften}

Universität Nantes (Frankreich) seit 11.05.1973

Universität Neapel (Italien) seit 24.04.1985

Universität Peking (China) seit 01.07.1987

Universität Alicante (Spanien) seit 01.12.1987

Universität Reading (Großbritannien) seit 13.12.1988

University of California, Davis (USA) seit 10.10.1990

Pennsylvania State University (USA) seit 05.12.1990

Karls-Universität Prag (Tschechische Republik) seit 18.03.1998

Keio University, Tokio (Japan) seit 04.02.1999 


\section{Personal}

\section{Universität}

\begin{tabular}{l|r|r|r} 
& $\begin{array}{l}\text { Professoren/ } \\
\text { Professorinnen }\end{array}$ & $\begin{array}{l}\text { wissensch. } \\
\text { MA }^{1}\end{array}$ & $\begin{array}{l}\text { weiteres } \\
\text { Personal }\end{array}$ \\
\hline Mathematisch-Naturw. Fakultät & 94 & 288 & 287 \\
Philosophische Fakultät & 60 & 117 & 48 \\
Juristische Fakultät & 15 & 44 & 13 \\
Wirtschaftswiss. Fakultät & 10 & 29 & 12 \\
Medizinische Fakultät & 80 & 998 & -2 \\
\hline Universitäts- und Landesbibliothek & - & - & $135^{3}$ \\
Zentrum für Informations- und & - & - & $45^{3}$ \\
Medientechnologie & - & - & $312^{3}$ \\
Verwaltung & $\mathbf{2 5 9}$ & $\mathbf{1 . 4 7 6}$ & $\mathbf{8 5 2}$
\end{tabular}

Auszubildende (ohne Medizin): 51

${ }^{1}$ MA: Mitarbeiter/-innen

${ }^{2}$ Das weitere Personal wird beim Universitätsklinikum geführt.

${ }^{3}$ Wissenschaftliches und Nichtwissenschaftliches Personal

\section{Universitätsklinikum}

Im Jahresdurchschnitt Beschäftigte

Mitarbeiter/-innen (Vollkräfte) ca.

darunter:

$\begin{array}{ll}\text { Wiss. Personal }^{1} & 776\end{array}$

$\begin{array}{lr}\text { Pflegedienst ca. } & 878\end{array}$

Medizinisch technischer Dienst ca. $\quad 1.130$

Personal in medizinnahen Berufen ca. $\quad 411$

Klinisches Hauspersonal $\quad 69$

sonstiges Personal (Verwaltung, Technik, Wirtschaft und 881

Versorgung, Sonderdienst, Personal in Ausbildungsstätten)

Ausbildungsplätze (Ausbildungszentrum für $\quad 430$

Gesundheitsberufe, Sonstige)

\begin{tabular}{lr}
\hline Anzahl Kliniken & 31 \\
Anzahl Institute & 31 \\
Betriebene Betten & 1.158 \\
Stationäre Behandlungen & 42.384 \\
Ambulante Behandlungen & 144.977
\end{tabular}

${ }^{1}$ In diesem Umfang ist das wissenschaftliche Personal der Medizinischen Fakultät verpflichtet, Aufgaben in der Krankenversorgung im Universitätsklinikum wahrzunehmen. Quelle: Jahresbericht Klinikum 2008 
Daten und Abbildungen aus dem Zahlenspiegel der Heinrich-Heine-Universität Düsseldorf 929

\section{Haushalt}

\begin{tabular}{l|r|r} 
& $\begin{array}{l}\text { Haushaltsvolumen } \\
\text { in 1000 Euro }\end{array}$ & $\begin{array}{l}\text { Drittmittelausgaben } \\
\text { in 1000 Euro }\end{array}$ \\
\hline Math.-Nat. Fakultät & 16.375 \\
$\begin{array}{l}\text { Philosophische Fakultät } \\
\text { Juristische Fakultät }\end{array}$ & 3.293 \\
Wirtschaftswiss. Fakultät & & 616 \\
\hline $\begin{array}{l}\text { Medizinische Fakultät } \\
\text { (Landeszuschuss) }\end{array}$ & $108.479^{2}$ & 73 \\
\hline $\begin{array}{l}\text { Heinrich-Heine-Universität } \\
\text { (Landeszuschuss) }\end{array}$ & $123.654^{3}$ & 28.659 \\
\hline Summe & $\mathbf{2 3 2 . 1 3 3}$ & $\mathbf{4 9 . 0 1 6}$ \\
${ }^{1}$ ohne Medizinische Fakultät \\
${ }^{2}$ Zuführungen für den laufenden Betrieb an die Fakultät \\
${ }^{3}$ incl. 33,3 Mio. Euro für Mieten und Pachten an den BLB NRW
\end{tabular}

Drittmittelausgaben je Fakultät im Haushaltsjahr 2008 in Euro

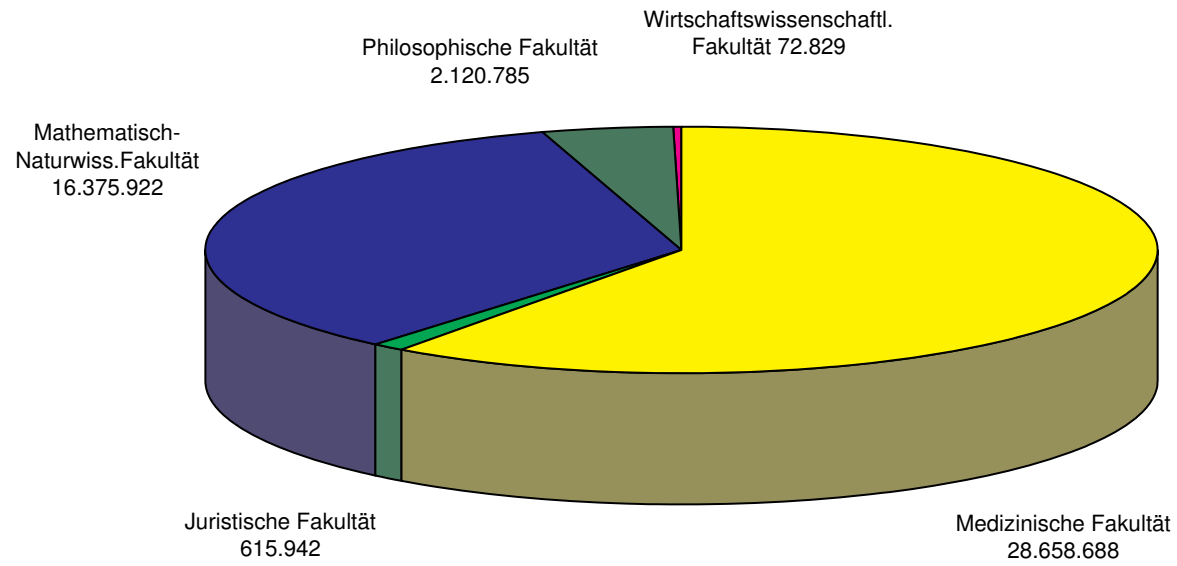


Studiengänge an der Heinrich-Heine-Universität Düsseldorf (Stand WS 2008/2009)

\begin{tabular}{|c|c|c|c|c|c|c|c|}
\hline Abschluss $\rightarrow$ & 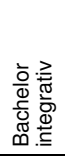 & 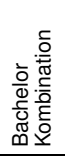 & 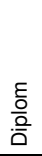 & 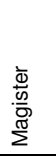 & 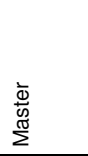 & 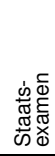 & $\begin{array}{l}\text { 듬 } \\
\text { 음 } \\
\text { 음 }\end{array}$ \\
\hline $\begin{array}{l}\text { Allgemeine Sprachwissenschaft } \\
\text { Anglistik / Englisch } \\
\text { Antike Kultur } \\
\text { Betriebswirtschaftslehre } \\
\text { Biochemie }\end{array}$ & $\stackrel{\bullet}{\bullet}$ & $\begin{array}{l}\bullet \\
\circ\end{array}$ & + & $\begin{array}{l}+ \\
+\end{array}$ & $\begin{array}{l}\bullet \\
\bullet\end{array}$ & & $\begin{array}{l}\bullet \\
\bullet \\
\bullet \\
\bullet\end{array}$ \\
\hline $\begin{array}{l}\text { Biologie } \\
\text { Chemie } \\
\text { Erziehungswissenschaft } \\
\text { Geographie } \\
\text { Germanistik }\end{array}$ & $\bullet$ & $\bullet$ & $\begin{array}{l}+ \\
+\end{array}$ & $\begin{array}{l}+ \\
+ \\
+\end{array}$ & $\begin{array}{l} \\
\bullet \\
\bullet\end{array}$ & & $\begin{array}{l} \\
\bullet \\
\bullet \\
\bullet \\
+ \\
-\end{array}$ \\
\hline $\begin{array}{l}\text { Geschichte } \\
\text { Gewerblicher Rechtsschutz } \\
\text { Griechisch } \\
\text { Informatik } \\
\text { Informationsrecht } \\
\text { Informationswissenschaft }\end{array}$ & • & . & & $\begin{array}{l}+ \\
\bullet^{2} \\
+ \\
\bullet^{2} \\
+\end{array}$ & • & & $\begin{array}{l}\bullet \\
\bullet \\
\bullet \\
\bullet\end{array}$ \\
\hline $\begin{array}{l}\text { Informationswiss. u. Sprachtechnologie } \\
\text { Jiddische Kultur, Sprache u. Literatur } \\
\text { Jiddistik } \\
\text { Jüdische Studien } \\
\text { Kommunikations- u. Medienwissenschaft }\end{array}$ & $\bullet$ & • & & $\begin{array}{l}+ \\
+\end{array}$ & $\begin{array}{l}\bullet \\
\bullet \\
\bullet\end{array}$ & & $\begin{array}{l}\bullet \\
\bullet \\
\bullet \\
\bullet\end{array}$ \\
\hline $\begin{array}{l}\text { Kunstgeschichte } \\
\text { Latein } \\
\text { Linguistik } \\
\text { Literaturübersetzen } \\
\text { Mathematik }\end{array}$ & $\bullet$ & $\circ$ & $\begin{array}{l}+ \\
+\end{array}$ & $\begin{array}{l}+ \\
+\end{array}$ & $\begin{array}{l}\bullet \\
\bullet \\
\bullet \\
\bullet \\
\bullet\end{array}$ & & $\begin{array}{l}\bullet \\
\bullet\end{array}$ \\
\hline $\begin{array}{l}\text { Medienkulturanalyse } \\
\text { Medienwissenschaft } \\
\text { Medien- und Kulturwissenschaft } \\
\text { Medizin } \\
\text { Medizinische Physik }\end{array}$ & • & & & & $\bullet$ & • & $\begin{array}{l}+ \\
+ \\
\bullet\end{array}$ \\
\hline $\begin{array}{l}\text { Medizinrecht } \\
\text { Modernes Japan } \\
\text { Musikwissenschaft } \\
\text { Pharmazie } \\
\text { Philosophie }\end{array}$ & & • & & $\begin{array}{l}+ \\
+ \\
+\end{array}$ & $\begin{array}{l}\bullet^{2} \\
\bullet \\
\bullet\end{array}$ & • & • \\
\hline $\begin{array}{l}\text { Physik } \\
\text { Politikwissenschaft } \\
\text { Politische Kommunikation } \\
\text { Psychologie } \\
\text { Public Health }\end{array}$ & • & $\circ$ & + & + & $\begin{array}{l}\bullet \\
\bullet \\
\bullet \\
\end{array}$ & & $\bullet \bullet$ \\
\hline $\begin{array}{l}\text { Recht für ausländische Juristen } \\
\text { Rechtswissenschaft } \\
\text { Romanistik } \\
\text { Sozialwissenschaften } \\
\text { Soziologie }\end{array}$ & • & $\circ$ & & $\begin{array}{l}++^{3} \\
+\end{array}$ & $\bullet$ & $\bullet$ & $\begin{array}{l}\bullet \\
\bullet \\
\bullet\end{array}$ \\
\hline $\begin{array}{l}\text { Sportwissenschaften } \\
\text { The Americas-Las Américas-Les Amériques } \\
\text { Wirtschaftschemie } \\
\text { Zahnmedizin }\end{array}$ & • & & + & & • & & + \\
\hline
\end{tabular}

- Hauptfach / Kernfach und Nebenfach / Ergänzungsfach, o nur Nebenfach / Ergänzungsfach,

+ Studiengang läuft aus, ${ }^{1}$ erstmals ab WS 2008/2009, ${ }^{2}$ Weiterbildungsstudiengang, ${ }^{3}$ Zusatzstudiengang 
Studierende (Studienfälle/Personen) aller Studienfächer/Studiengangkombinationen WS 2008/2009 (Quelle: Dezernat 1)

\begin{tabular}{|c|c|c|c|c|c|c|c|c|}
\hline Abschluss $\rightarrow$ & 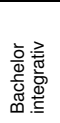 & 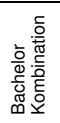 & $\begin{array}{l}\text { E } \\
\text { 응 } \\
\overline{0}\end{array}$ & 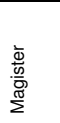 & 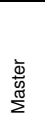 & 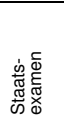 & $\begin{array}{l}\text { 듬 } \\
\text { 흠 } \\
\text { 홈 }\end{array}$ & है \\
\hline Allgemeine Sprachwissenschaft & 0 & 0 & 0 & 278 & 0 & 0 & 12 & 290 \\
\hline Anglistik/Englisch & 0 & 694 & 0 & 753 & 34 & 0 & 60 & 1.514 \\
\hline Antike Kultur & 0 & 226 & 0 & 0 & 0 & 0 & 0 & 226 \\
\hline Erziehungswissenschaft & 0 & 0 & 8 & 19 & 0 & 0 & 27 & 54 \\
\hline Germanistik & 0 & 1.026 & 0 & 1.870 & 50 & 0 & 151 & 3.097 \\
\hline Geschichte & 0 & 984 & 0 & 572 & 20 & 0 & 101 & 1.677 \\
\hline Griechisch & 0 & 0 & 0 & 11 & 0 & 0 & 2 & 13 \\
\hline Informationswissenschaft & 0 & 48 & 0 & 186 & 0 & 0 & 24 & 258 \\
\hline Informationswiss. und Sprachtechnologie & 94 & 0 & 0 & 0 & 2 & 0 & 0 & 96 \\
\hline Jiddische Kultur, Sprache und Literatur & 0 & 40 & 0 & 11 & 0 & 0 & 0 & 51 \\
\hline Jiddistik & 0 & 0 & 0 & 0 & 0 & 0 & 0 & 0 \\
\hline Jüdische Studien & 0 & 45 & 0 & 26 & 1 & 0 & 2 & 74 \\
\hline Kommunikations- und Medienwissenschaft & 0 & 110 & 0 & 0 & 0 & 0 & 7 & 117 \\
\hline Kunstgeschichte & 0 & 303 & 0 & 94 & 26 & 0 & 39 & 462 \\
\hline Latein & 0 & 0 & 0 & 23 & 0 & 0 & 3 & 26 \\
\hline Linguistik & 88 & 71 & 0 & 0 & 12 & 0 & 0 & 171 \\
\hline Literaturübersetzen & 0 & 0 & 115 & 0 & 5 & 0 & 0 & 120 \\
\hline Medien- und Kulturwissenschaft & 234 & 0 & 0 & 0 & 0 & 0 & 9 & 243 \\
\hline Medienkulturanalyse & 0 & 0 & 0 & 0 & 89 & 0 & 0 & 89 \\
\hline Medienwissenschaft & 0 & 0 & 0 & 125 & 0 & 0 & 17 & 142 \\
\hline Modernes Japan & 0 & 356 & 0 & 111 & 3 & 0 & 10 & 480 \\
\hline Musikwissenschaft & 0 & 116 & 0 & 35 & 0 & 0 & 0 & 151 \\
\hline Philosophie & 0 & 552 & 0 & 267 & 7 & 0 & 42 & 868 \\
\hline Politikwissenschaft & 0 & 106 & 0 & 176 & 0 & 0 & 24 & 306 \\
\hline Politische Kommunikation & 0 & 0 & 0 & 0 & 44 & 0 & 0 & 44 \\
\hline Psychologie* $^{*}$ & 0 & 0 & 0 & 4 & 0 & 0 & 7 & 11 \\
\hline Romanistik & 0 & 496 & 0 & 298 & 4 & 0 & 36 & 834 \\
\hline Sozialwissenschaften & 329 & 0 & 0 & 0 & 53 & 0 & 2 & 384 \\
\hline Soziologie & 0 & 103 & 0 & 138 & 0 & 0 & 13 & 254 \\
\hline Sportwissenschaft & 0 & 0 & 0 & 0 & 0 & 0 & 4 & 4 \\
\hline The Americas-Las Américas-Les Amériques & 0 & 0 & 0 & 0 & 14 & 0 & 0 & 14 \\
\hline Philosophische Fakultät & 745 & 5.276 & 123 & 4.997 & 364 & 0 & 592 & 12.097 \\
\hline Rechtswissenschaft & 0 & 0 & 0 & 0 & 0 & 1.188 & 176 & 1.364 \\
\hline Recht f. ausländische Juristen & 0 & 0 & 0 & 3 & 0 & 0 & 0 & 3 \\
\hline Juristische Fakultät & 0 & 0 & 0 & 3 & 0 & 1.188 & 176 & 1.367 \\
\hline Betriebswirtschaftslehre & 460 & 0 & 481 & 0 & 0 & 0 & 28 & 969 \\
\hline Wirtschaftswissenschaftliche Fakultät & 460 & 0 & 481 & 0 & 0 & 0 & 28 & 969 \\
\hline Biochemie & 92 & 0 & 0 & 0 & 36 & 0 & 16 & 144 \\
\hline Biologie & 463 & 0 & 647 & 0 & 11 & 0 & 279 & 1.400 \\
\hline Chemie & 177 & 0 & 234 & 0 & 5 & 0 & 138 & 554 \\
\hline Geographie & 0 & 0 & 0 & 18 & 0 & 0 & 10 & 28 \\
\hline Informatik & 514 & 0 & 0 & 0 & 71 & 0 & 37 & 622 \\
\hline Mathematik & 205 & 0 & 165 & 0 & 22 & 0 & 30 & 422 \\
\hline Medizinische Physik & 65 & 0 & 0 & 0 & 0 & 0 & 0 & 65 \\
\hline Pharmazie & 0 & 0 & 0 & 0 & 0 & 552 & 60 & 612 \\
\hline Physik & 158 & 0 & 70 & 0 & 16 & 0 & 43 & 287 \\
\hline Psychologie & 194 & 0 & 283 & 0 & 0 & 0 & 58 & 535 \\
\hline Wirtschaftschemie ${ }^{\star \star}$ & 120 & 0 & 148 & 0 & 0 & 0 & 6 & 274 \\
\hline Mathematisch-Naturwiss. Fakultät & 1.988 & 0 & 1.547 & 18 & 161 & 552 & 677 & 4.943 \\
\hline Medizin & 0 & 0 & 0 & 0 & 0 & 2.435 & 75 & 2.510 \\
\hline Public Health & 0 & 0 & 0 & 42 & 0 & 0 & 5 & 47 \\
\hline Zahnmedizin & 0 & 0 & 0 & 0 & 0 & 351 & 19 & 370 \\
\hline Medizinische Fakultät & 0 & 0 & 0 & 42 & 0 & 2.786 & 99 & 2.927 \\
\hline Hochschule gesamt & 3.193 & 5.276 & 2.151 & 5.060 & 525 & 4.526 & 1.572 & 22.303 \\
\hline Personen & 3.101 & 2.582 & 2.073 & 1.717 & 510 & 4.505 & 1.443 & 15.931 \\
\hline
\end{tabular}

Außerdem 107 Austauschstudierende, 17 Studierende ohne angestrebten Abschluss (19 FF), 378 Beurlaubte (582 FF), 402 Gasthörer (450 FF) und 30 Besucher Deutschkurs (30 FF). * Magister Nebenfach. ** Gemeinschaftlicher Studiengang von Wirtschaftswissenschaftlicher und MathematischNaturwissenschaftlicher Fakultät. 
Ausländische Studierende nach Herkunftsländern seit WS 2003/2004 (Quelle: Dezernat 1)

\begin{tabular}{|c|c|c|c|c|c|}
\hline Herkunftsländer & $\begin{array}{c}\text { WS } \\
2004 / 2005\end{array}$ & $\begin{array}{c}\text { WS } \\
2005 / 2006\end{array}$ & $\begin{array}{c}\text { WS } \\
2006 / 2007\end{array}$ & $\begin{array}{c}\text { WS } \\
2007 / 2008\end{array}$ & $\begin{array}{c}\text { WS } \\
2008 / 2009\end{array}$ \\
\hline Türkei & 305 & 307 & 315 & 266 & 267 \\
\hline Russische Föderation & 165 & 172 & 174 & 169 & 167 \\
\hline Polen & 199 & 195 & 188 & 151 & 131 \\
\hline Ukraine & 128 & 132 & 140 & 129 & 125 \\
\hline Bulgarien & 131 & 147 & 154 & 135 & 118 \\
\hline Griechenland & 138 & 136 & 132 & 122 & 117 \\
\hline Marokko & 137 & 149 & 163 & 143 & 115 \\
\hline Georgien & 90 & 106 & 98 & 106 & 108 \\
\hline China (VR) (einschl. Tibet) & 131 & 125 & 132 & 113 & 104 \\
\hline Iran & 89 & 88 & 86 & 76 & 83 \\
\hline Italien & 71 & 64 & 69 & 66 & 62 \\
\hline Tunesien & 24 & 30 & 46 & 52 & 55 \\
\hline Süd Korea & 75 & 61 & 69 & 62 & 54 \\
\hline Jugoslawien (Serbien, Montenegro) & 82 & 80 & 74 & 44 & 50 \\
\hline Kamerun & 48 & 52 & 56 & 54 & 48 \\
\hline Bosnien-Herzegowina & 49 & 50 & 49 & 41 & 47 \\
\hline Kroatien & 71 & 65 & 62 & 59 & 46 \\
\hline Syrien & 28 & 33 & 36 & 34 & 39 \\
\hline Weissrussland (Belarus) & 33 & 33 & 31 & 35 & 31 \\
\hline Rumänien & 28 & 35 & 35 & 29 & 29 \\
\hline Spanien & 47 & 38 & 35 & 29 & 29 \\
\hline Mazedonien & 26 & 26 & 23 & 27 & 24 \\
\hline Litauen & 22 & 22 & 22 & 18 & 24 \\
\hline Japan & 40 & 29 & 27 & 24 & 23 \\
\hline Israel & 26 & 23 & 25 & 24 & 23 \\
\hline Indien & 32 & 32 & 31 & 23 & 21 \\
\hline Portugal & 15 & 17 & 21 & 19 & 21 \\
\hline Kenia & 5 & 8 & 10 & 14 & 20 \\
\hline Vietnam & 12 & 13 & 21 & 21 & 18 \\
\hline Österreich & 19 & 22 & 19 & 18 & 18 \\
\hline Niederlande & 21 & 20 & 21 & 17 & 18 \\
\hline Großbritannien & 35 & 29 & 24 & 16 & 16 \\
\hline Albanien & 9 & 13 & 11 & 10 & 16 \\
\hline Moldau, Republik & 13 & 15 & 15 & 18 & 15 \\
\hline Ägypten & 14 & 16 & 16 & 14 & 15 \\
\hline Mongolei & 21 & 19 & 15 & 13 & 14 \\
\hline Usbekistan & 6 & 7 & 8 & 11 & 14 \\
\hline Indonesien & 9 & 14 & 11 & 15 & 13 \\
\hline Kasachstan & 10 & 10 & 14 & 11 & 13 \\
\hline Frankreich & 35 & 29 & 17 & 16 & 12 \\
\hline Libanon & 7 & 7 & 9 & 14 & 12 \\
\hline Irak & 10 & 11 & 14 & 12 & 12 \\
\hline Estland & 13 & 16 & 15 & 11 & 12 \\
\hline Brasilien & 13 & 13 & 13 & 12 & 11 \\
\hline Ungarn & 28 & 20 & 17 & 14 & 10 \\
\hline USA & 14 & 12 & 13 & 13 & 10 \\
\hline Senegal & 13 & 17 & 19 & 12 & 10 \\
\hline Kirgisien & 8 & 11 & 10 & 11 & 10 \\
\hline Luxemburg & 12 & 9 & 9 & 11 & 10 \\
\hline sonstige $^{1}$ & 260 & 251 & 262 & 248 & 265 \\
\hline Summe & 2.817 & 2.829 & 2.876 & 2.602 & 2.525 \\
\hline
\end{tabular}

Nur Studierende mit angestrebtem Abschluss, ohne Gasthörer, Beurlaubte und Besucher Deutschkurs. ${ }^{1}$ Zusammenfassung von Herkunftsländern, denen im WS 2008/2009 weniger als 10 Studierende angehörten, sowie von Staatenlosen und sonstigen Fällen. 
Daten und Abbildungen aus dem Zahlenspiegel der Heinrich-Heine-Universität Düsseldorf 933

Studierende insgesamt (Personen) im ersten Studienfach in den Fakultäten, WS 2008/2009

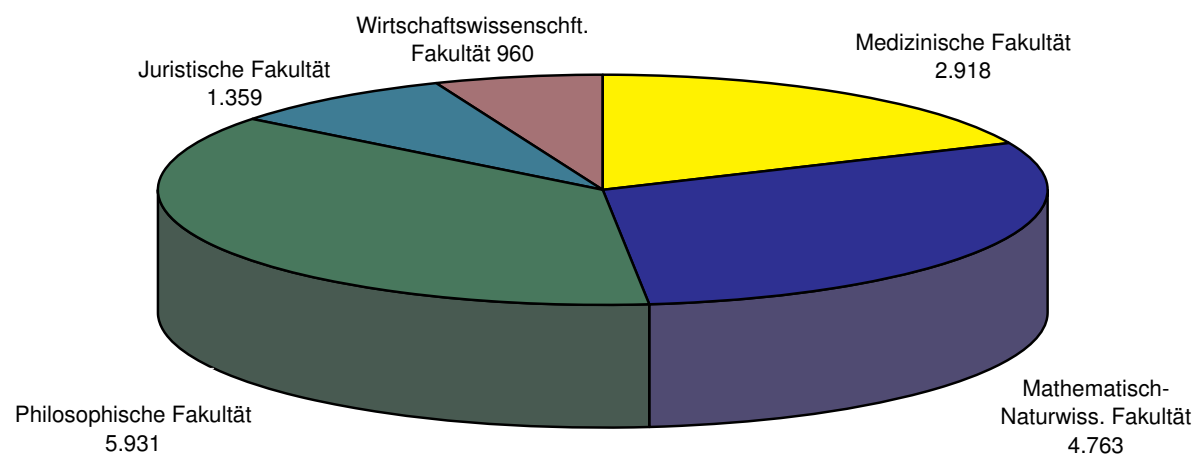

Studienanfänger/innen (Personen) im ersten FS in den Fakultäten WS 2008/2009

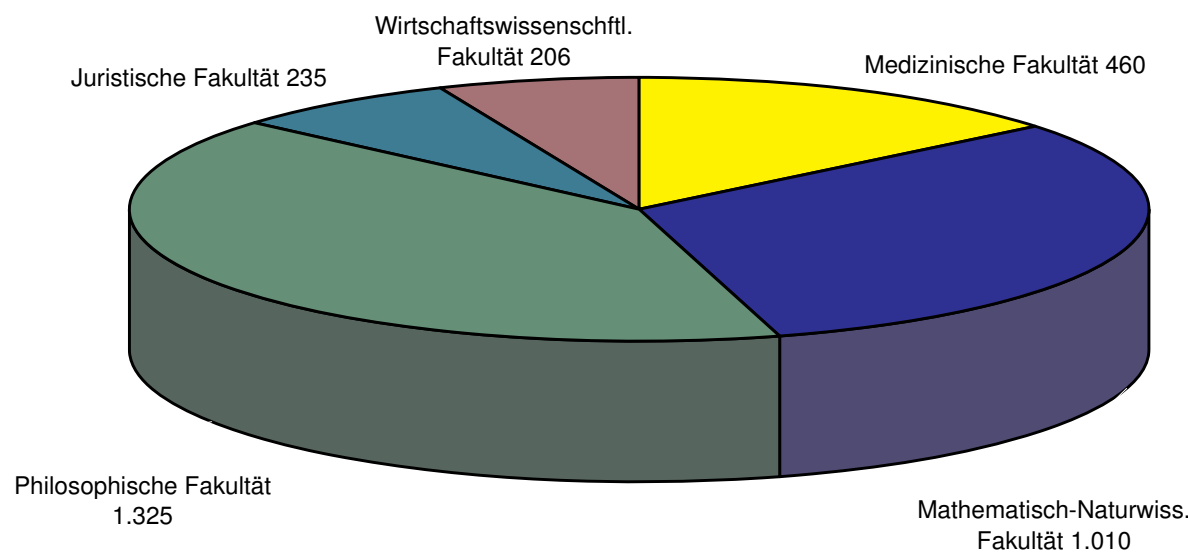


Studienabschlüsse (Prüfungen/Personen) im Studienjahr 2008 (Quelle: Prüfungsamt)

\begin{tabular}{|c|c|c|c|c|c|c|c|c|}
\hline Abschluss $\rightarrow$ & 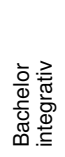 & 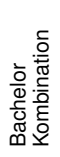 & $\begin{array}{l}\varepsilon \\
\frac{\mathrm{o}}{0} \\
\frac{\mathrm{O}}{0}\end{array}$ & $\begin{array}{l}\frac{\bar{\Phi}}{0} \\
\frac{0}{0} \\
\frac{\pi}{\Sigma}\end{array}$ & $\begin{array}{l}\frac{\bar{d}}{w} \\
\frac{\pi}{\pi} \\
\sum\end{array}$ & 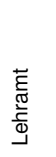 & 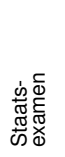 & $\begin{array}{l}\stackrel{\Phi}{E} \\
\text { है } \\
\text { ڤ }\end{array}$ \\
\hline Allgemeine Sprachwissenschaft & 0 & 0 & 0 & 32 & 0 & 0 & 0 & 32 \\
\hline Anglistik/Englisch & 0 & 25 & 0 & 164 & 3 & 17 & 0 & 209 \\
\hline Antike Kultur & 0 & 3 & 0 & 0 & 0 & 0 & 0 & 3 \\
\hline Erziehungswissenschaft & 0 & 0 & 11 & 30 & 0 & 6 & 0 & 47 \\
\hline Germanistik/Deutsch & 0 & 48 & 0 & 379 & 3 & 20 & 0 & 450 \\
\hline Geschichte & 0 & 34 & 0 & 98 & 6 & 8 & 0 & 146 \\
\hline Griechisch & 0 & 0 & 0 & 1 & 0 & 0 & 0 & 1 \\
\hline Informationswissenschaft & 0 & 5 & 0 & 68 & 0 & 0 & 0 & 73 \\
\hline Informationswiss. u. Sprachtechnologie & 5 & 0 & 0 & 0 & 0 & 0 & 0 & 5 \\
\hline Jiddische Kultur, Sprache u. Literatur & 0 & 1 & 0 & 2 & 0 & 0 & 0 & 3 \\
\hline Jiddistik & 0 & 0 & 0 & 0 & 0 & 0 & 0 & 0 \\
\hline Jüdische Studien & 0 & 2 & 0 & 3 & 0 & 0 & 0 & 5 \\
\hline Kommunikations- u. Medienwissenschaft & 0 & 27 & 0 & 0 & 0 & 0 & 0 & 27 \\
\hline Kunstgeschichte & 0 & 15 & 0 & 17 & 9 & 5 & 0 & 46 \\
\hline Latein & 0 & 0 & 0 & 0 & 0 & 1 & 0 & 1 \\
\hline Linguistik & 18 & 9 & 0 & 0 & 1 & 0 & 0 & 28 \\
\hline Literaturübersetzen & 0 & 0 & 15 & 0 & 0 & 0 & 0 & 15 \\
\hline Medienwissenschaft & 0 & 0 & 0 & 45 & 0 & 0 & 0 & 45 \\
\hline Medien- $\mathrm{u}$. Kulturwissenschaft & 56 & 0 & 0 & 0 & 0 & 0 & 0 & 56 \\
\hline Medienkulturanalyse & 0 & 0 & 0 & 0 & 27 & 0 & 0 & 27 \\
\hline Modernes Japan & 0 & 14 & 0 & 17 & 0 & 0 & 0 & 31 \\
\hline Musikwissenschaft & 0 & 1 & 0 & 6 & 0 & 0 & 0 & 7 \\
\hline Philosophie & 0 & 12 & 0 & 33 & 2 & 9 & 0 & 56 \\
\hline Politikwissenschaft & 0 & 21 & 0 & 43 & 0 & 0 & 0 & 64 \\
\hline Politische Kommunikation & 0 & 0 & 0 & 0 & 11 & 0 & 0 & 11 \\
\hline Psychologie* & 0 & 0 & 0 & 5 & 0 & 0 & 0 & 5 \\
\hline Romanistik/Französisch/Italienisch/Spanisch & 0 & 20 & 0 & 31 & 0 & 17 & 0 & 68 \\
\hline Sozialwissenschaften & 109 & 0 & 0 & 0 & 18 & 0 & 0 & 127 \\
\hline Soziologie & 0 & 7 & 0 & 31 & 0 & 1 & 0 & 39 \\
\hline Sport & 0 & 0 & 0 & 3 & 0 & 14 & 0 & 17 \\
\hline Philosophische Fakultät & 188 & 244 & 26 & 1.008 & 80 & 98 & 0 & 1.644 \\
\hline Recht f. ausländische Juristen & 0 & 0 & 0 & 4 & 0 & 0 & 0 & 4 \\
\hline Rechtswissenschaft & 0 & 0 & 0 & 0 & 0 & 0 & 105 & 105 \\
\hline Juristische Fakultät & 0 & 0 & 0 & 4 & 0 & 0 & 105 & 109 \\
\hline Betriebswirtschaftslehre & 0 & 0 & 129 & 0 & 0 & 0 & 0 & 129 \\
\hline Wirtschaftswissenschaftliche Fakultät & 0 & 0 & 129 & 0 & 0 & 0 & 0 & 129 \\
\hline Biochemie & 15 & 0 & 0 & 0 & 14 & 0 & 0 & 29 \\
\hline Biologie & 6 & 0 & 121 & 0 & 1 & 11 & 0 & 139 \\
\hline Chemie & 5 & 0 & 40 & 0 & 0 & 4 & 0 & 49 \\
\hline Geographie & 0 & 0 & 0 & 8 & 0 & 2 & 0 & 10 \\
\hline Informatik & 38 & 0 & 0 & 0 & 23 & 0 & 0 & 61 \\
\hline Mathematik & 17 & 0 & 23 & 0 & 5 & 4 & 0 & 49 \\
\hline Pharmazie & 0 & 0 & 0 & 0 & 0 & 0 & 102 & 102 \\
\hline Physik & 10 & 0 & 19 & 0 & 1 & 5 & 0 & 35 \\
\hline Psychologie & 0 & 0 & 36 & 0 & 0 & 0 & 0 & 36 \\
\hline Wirtschaftschemie ${ }^{* \star}$ & 0 & 0 & 29 & 0 & 0 & 0 & 0 & 29 \\
\hline Mathematisch-Naturwiss. Fakultät & 91 & 0 & 268 & 8 & 44 & 26 & 102 & 539 \\
\hline Public Health & 0 & 0 & 0 & 19 & 0 & 0 & 0 & 19 \\
\hline Medizin & 0 & 0 & 0 & 0 & 0 & 0 & 240 & 240 \\
\hline Zahnmedizin & 0 & 0 & 0 & 0 & 0 & 0 & 48 & 48 \\
\hline Medizinische Fakultät & 0 & 0 & 0 & 19 & 0 & 0 & 288 & 307 \\
\hline Hochschule gesamt & 279 & 244 & 423 & 1.039 & 124 & 124 & 495 & 2.728 \\
\hline Personen & 279 & 122 & 423 & 375 & 124 & 62 & 495 & 1.880 \\
\hline
\end{tabular}

* Magister Nebenfach. ** Gemeinschaftlicher Studiengang von Wirtschaftswissenschaftlicher und MathematischNaturwissenschaftlicher Fakultät. 
Daten und Abbildungen aus dem Zahlenspiegel der Heinrich-Heine-Universität Düsseldorf 935

Studienabschlüsse in den Fakultäten Studienjahr 2008

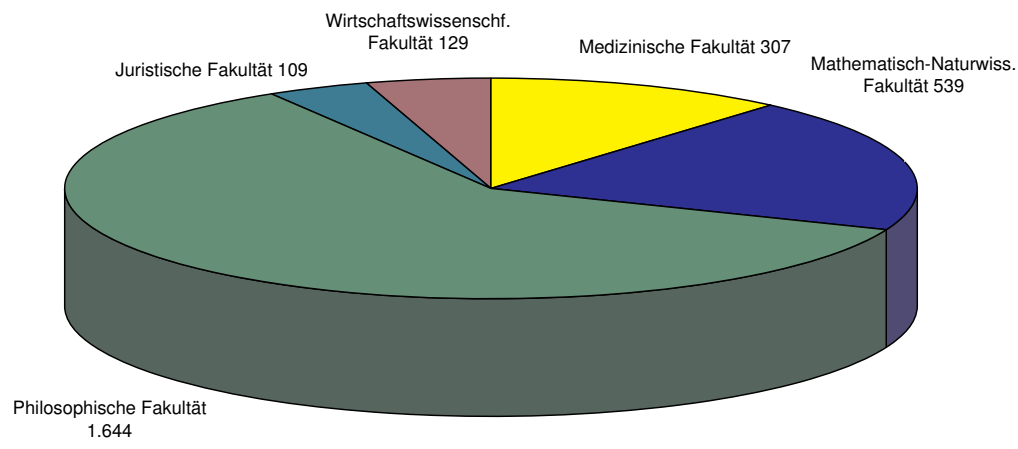

Studienabschlüsse nach Abschlussarten, Studienjahr 2008

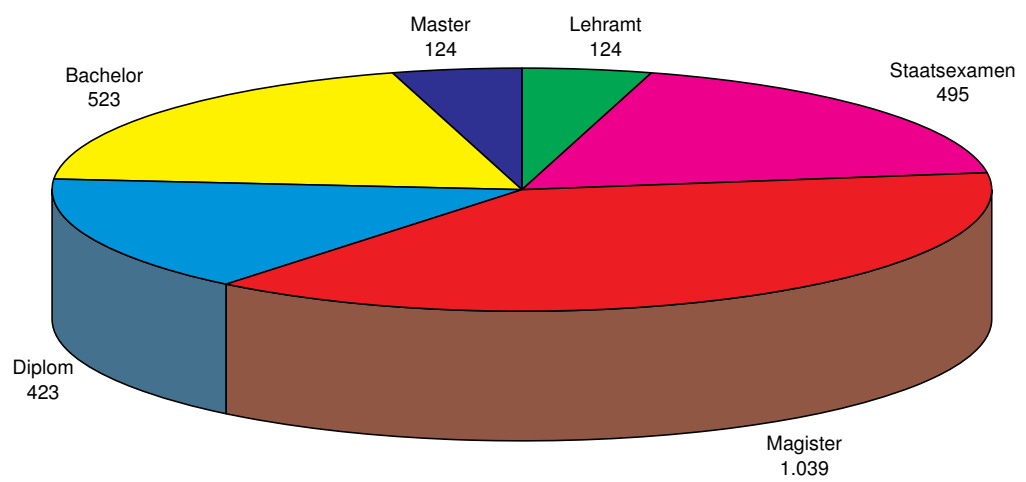

\section{Habilitationen und Promotionen im Studienjahr 2008}

Studienjahr 2008

\begin{tabular}{lcr} 
& Habilitationen & Promotionen \\
\hline Math.-Nat. Fakultät & 6 & 99 \\
Philosophische & 5 & 27 \\
Fakultät & & \\
Juristische Fakultät & 0 & 19 \\
Wirtschaftswiss. & 0 & 5 \\
Fakultät & & \\
Medizinische Fakultät & 17 & 128 \\
\hline Summe & $\mathbf{2 8}$ & $\mathbf{2 7 8}$
\end{tabular}

(Studienjahr: WS 2007/2008 u. SS 2008) 
Universitäts- und Landesbibliothek

\begin{tabular}{|l|r|r|r|r|r|}
\hline & $2003 / 2004$ & $2004 / 2005$ & \multicolumn{1}{|c|}{2006} & 2007 & 2007 \\
\hline Aktive Benutzerinnen und Benutzer & 25.043 & 22.937 & 21.842 & 21.911 & 22.975 \\
Besucherinnen und Besucher & 854.698 & 849.026 & 1.239 .857 & 1.882 .771 & 1.990 .903 \\
Öffnungsstunden pro Woche & 81 & 88 & 88 & 110 & 110 \\
Ausleihen & 1.306 .006 & 1.399 .299 & 1.405 .088 & 1.154 .189 & 1.061 .237 \\
\hline Vormerkungen & 79.455 & 53.070 & 45.481 & 44.570 & 40.221 \\
Versandte Fernleihen & 30.613 & 32.208 & 39.827 & 37.254 & 35.656 \\
Erhaltene Fernleihen & 32.532 & 29.475 & 40.026 & 36.102 & 33.366 \\
Gesamtbestand nach Buchbindereinheiten & 2.496 .906 & 2.494 .906 & 2.467 .861 & 2.411 .832 & 2.399 .451 \\
\hline Neuzugang nach Bänden & 32.580 & 34.748 & 34.841 & 37.494 & 43.511 \\
laufend gehaltene gedruckte Zeitschriften & 6.128 & 5.539 & 4.819 & 4.242 & 4.127 \\
laufend gehaltene elektronische Zeitschriften & 4.580 & 5.249 & 6.724 & 9.294 & 12.010 \\
abbestellte Zeitschriften & 391 & 548 & 585 & 104 & 157 \\
\hline Lizenzierte Datenbanken & 47 & 384 & 703 & 404 & 500 \\
Zugriffe auf die Homepage & 3.610 .404 & 4.413 .007 & 18.985 .317 & 1.116 .511 & 2.451 .429 \\
Besuche Online-Katalog & 611.758 & 604.100 & 680.598 & 2.360 .890 & 3.033 .516 \\
Subskribenden Newsletter & 2.017 & 1.895 & 1.737 & 1.682 & 1.633 \\
\hline Schulungsstunden für Nutzerinnen und Nutzer & 69 & 98 & 303 & 435 & 397 \\
Zahl der Teilnehmenden an Schulungen & 1.068 & 3.228 & 6.444 & 5.145 & 7.265 \\
Ausstellungen & 6 & 5 & 5 & 5 & 5 \\
Publikationen der ULB & 12 & 15 & 22 & 11 & 12 \\
\hline
\end{tabular}

\section{ZIM - Zentrum für Informations- und Medientechnologie}

\begin{tabular}{|c|c|c|c|c|c|}
\hline & 2004 & 2005 & 2006 & 2007 & 2008 \\
\hline Benutzerinnen und Benutzer & 16.821 & 16.842 & 24.553 & 23.728 & 23.029 \\
\hline E-Mails pro Tag & 80.000 & 95.000 & 204.800 & 840.000 & 520.000 \\
\hline virenverseuchte E-Mails pro Tag ${ }^{1}$ & 2.000 & 6.800 & 2.000 & 960 & 1.344 \\
\hline Spam-E-Mails pro $\operatorname{Tag}^{1}$ & * & 68.000 & 150.000 & 750.000 & 490.500 \\
\hline Web-Zugriffe pro Tag & 771.500 & 920.068 & 1.044 .785 & 1.139 .051 & 1.226 .681 \\
\hline Netzanschlüsse auf dem Campus & 12.433 & 13.358 & 14.020 & 15.230 & 16.096 \\
\hline Switches/Router (Ports) & 7.148 & 7.710 & 8.201 & 9.306 & 10.175 \\
\hline WLan Access-Points & 50 & 53 & 53 & 80 & 128 \\
\hline Studierenden-Arbeitsplätze im ZIM & 261 & 261 & 261 & 263 & 247 \\
\hline Chipkarten für Druckdienst & 1.600 & * & * & * & * \\
\hline Druckseiten & 203.534 & * & * & * & * \\
\hline Posterdruck, Laminieren & 2.282 & 2.654 & 2.700 & 2.700 & 3.147 \\
\hline Zahl der Lehrveranstaltungen & 94 & 95 & 83 & 79 & * \\
\hline Zahl der Teilnehmenden & 2.012 & 2.021 & 1.587 & 1.217 & * \\
\hline durchgeführte Tagungen/Kongresse & 3 & 2 & * & * & * \\
\hline Zahl der Teilnehmenden & 550 & 370 & * & * & * \\
\hline Pools von Ausleihgeräten: & & & & & \\
\hline Notebooks & 52 & 47 & 50 & 67 & 71 \\
\hline Notebooks (Projekt Notebook-Univ.) & 42 & 42 & 42 & * & * \\
\hline Beamer & 65 & 47 & 47 & 42 & 47 \\
\hline sonstige Geräte & & 100 & 100 & 107 & 31 \\
\hline Zahl der Ausleihen (Beamer, Notebooks, & & & & & \\
\hline Recorder, Kameras, Audiogeräte) & 8.058 & 10.661 & 19.476 & * & $\begin{array}{r}19.866 \\
724\end{array}$ \\
\hline
\end{tabular}

* Einige Kennzahlen wurden neu aufgenommen, andere hingegen nicht mehr ausgewertet.

1 Zahlen für 2006 geschätzt. 